\title{
Semiparametric Regression Analysis of Panel Count Data: A Practical Review
}

\author{
Sy Han Chiou ${ }^{1}{ }^{\oplus}$, Chiung-Yu Huang ${ }^{2}$, Gongjun $\mathrm{Xu}^{3}$ and \\ Jun $\operatorname{Yan}^{4}$
}

\author{
${ }^{1}$ Department of Mathematical Sciences, University of Texas at Dallas, USA \\ ${ }^{2}$ Department of Epidemiology and Biostatistics, University of California at San Francisco, USA \\ ${ }^{3}$ Department of Statistics, University of Michigan, USA \\ ${ }^{4}$ Department of Statistics, University of Connecticut, USA \\ E-mail:schiou@utdallas.edu
}

\begin{abstract}
Summary
Panel count data arise in many applications when the event history of a recurrent event process is only examined at a sequence of discrete time points. In spite of the recent methodological developments, the availability of their software implementations has been rather limited. Focusing on a practical setting where the effects of some time-independent covariates on the recurrent events are of primary interest, we review semiparametric regression modelling approaches for panel count data that have been implemented in $R$ package spef. The methods are grouped into two categories depending on whether the examination times are associated with the recurrent event process after conditioning on covariates. The reviewed methods are illustrated with a subset of the data from a skin cancer clinical trial.
\end{abstract}

Key words: Counting process; estimating equation; frailty; maximum likelihood; recurrent event.

\section{Introduction}

Panel count data are a special kind of event history data where the occurrence of recurrent events is observed only at a sequence of discrete time points, as opposed to being observed continuously in time. In contrast to conventional recurrent event data, where the exact occurrence times of the events are known, panel count data only have the count of events in each 'panel' between successive examination times points (Kalbfleisch \& Lawless, 1985). Panel count data frequently arise in many fields such as clinical trials, epidemiological studies and engineering, when continuous follow-up to obtain exact event times of each subject is infeasible or too costly. The term 'panel count' in econometrics refers to longitudinal or clustered count data (e.g. Riphahn et al., 2003;Croissant et al., 2008;Hsiao, 2014); although somewhat related, it is to be distinguished from the context of event history data as we focus on here.

The goal of this article is to review regression analysis for panel count data with a focus on methods that are available in the $\mathrm{R}$ environment (R Core Team, 2017). Many statistical methods 
have been developed to analyse panel count data, but quality controlled software implementation remains rather limited. In their recently published book on panel count data analysis, Sun \& Zhao (2013) noted the absence of actively maintained software packages at the time of writing their book (Sun \& Zhao, 2013, p.222). Two R packages for panel count data are publicly available at this time. Package spef (Chiou et al., 2017) provides multiple methods in a unified interface, with an earlier version presented in Wang \& Yan (2011). Package PCDSpline (Yao \& Wang, 2014) is an implementation of the gamma frailty model of (Yao et al., 2016). Instead of providing a comprehensive review of all existing methods, we focus on semiparametric regression models with time-independent covariates as implemented in the spef package; methods and software for handling time-varying covariates have been much less developed (Huang et al., 2010). Covariate effects on the recurrent events are of primary interest. Nonparametric estimation is possible with spef package by specifying an intercept-only model. We give more details on methods that are available in spef package and that were not treated in detail in Sun \& Zhao (2013). The illustration code will help readers who need to analyse a panel count dataset to obtain some quick insights easily.

One challenge in practical panel count data analysis is that the examination process or the follow-up time may be informative about the recurrent event process even after conditioning on available covariates. For example, patients with higher tumour recurrence rates may have more frequent clinical examinations as they may require more medical attention (Li et al., 2011; Sun \& Zhao, 2013). Another example is in labour progression of women giving childbirth, if each $1 \mathrm{~cm}$ increment of cervical dilation is treated as a recurrent event, then women with faster cervix dilation may have more frequent vaginal examinations (Ma \& Sundaram, 2018). Informative examination times are often encountered in panel count data, and falsely treating informative examination times as noninformative could result in biased regression coefficient estimation and misleading conclusions. Similar situations may arise where the follow-up time is informative. Therefore, we grouped the methods into two categories depending on whether or not informative examinations or follow-up times can be accommodated.

This article is organised as follows. A subset of the data from a skin tumour clinical trial is introduced in Section 2 to demonstrate the structure and graphical features of panel count data. Notations of observed data and some of the most popular semiparametric models are presented in Section 3. Methods under the assumption of noninformative and informative examination/censoring times are reviewed in Sections 4 and 5, respectively, illustrated with the skin tumour data. The performances of the implemented methods under different settings in a simulation study are reported in Section 6. A discussion concludes in Section 7.

\section{Skin Cancer Chemoprevention Trail}

We illustrate the usage of the spef package with a skin cancer prevention study (Bailey et al., 2010). The whole dataset is available in Sun \& Zhao (2013, Table A.3.) and is included in the spef package under the name skinTumour. The study was a randomised, double-blind, placebo-controlled phase-3 clinical trial conducted at the University of Wisconsin Comprehensive Cancer Center. The primary objective was to determine whether the application of difluoromethylornithine (DFMO) as a chemoprevention agent would lead to a significant reduction in the occurrence of new skin tumours. The study consisted of 290 patients with a history of skin tumour. These patients were randomly assigned into two groups: a treatment group with oral DFMO at a daily dose of $0.5 \mathrm{gram} / \mathrm{m}^{2}$ and a placebo group with matching dosage. At each examination time during the follow-up, the number of newly developed skin tumours 
were counted, measured and removed. Comprehensive analysis of the whole data can be found in recent publications (e.g. Li et al., 2011;Sun \& Zhao, 2013; Chiou et al., 2017).

For illustration propose, we only use a subset of skinTumour containing 73 patients who enrolled in the study after the age of 70 years because some methods with bootstrapping are computationally demanding for large samples. Of the 73 patients, 40 were male and 41 were in the treatment group. The average number of examination times was 8.9 in this subset of patients, with three quartiles being 7,9 and 10. The average number of skin tumours developed for each patient in this subset throughout the study was 2.9 (median $=3$ ). We named this subset skiTum and used this name in the sequel. To view the structure of panel count data, we show the data for one patient (with id 10):

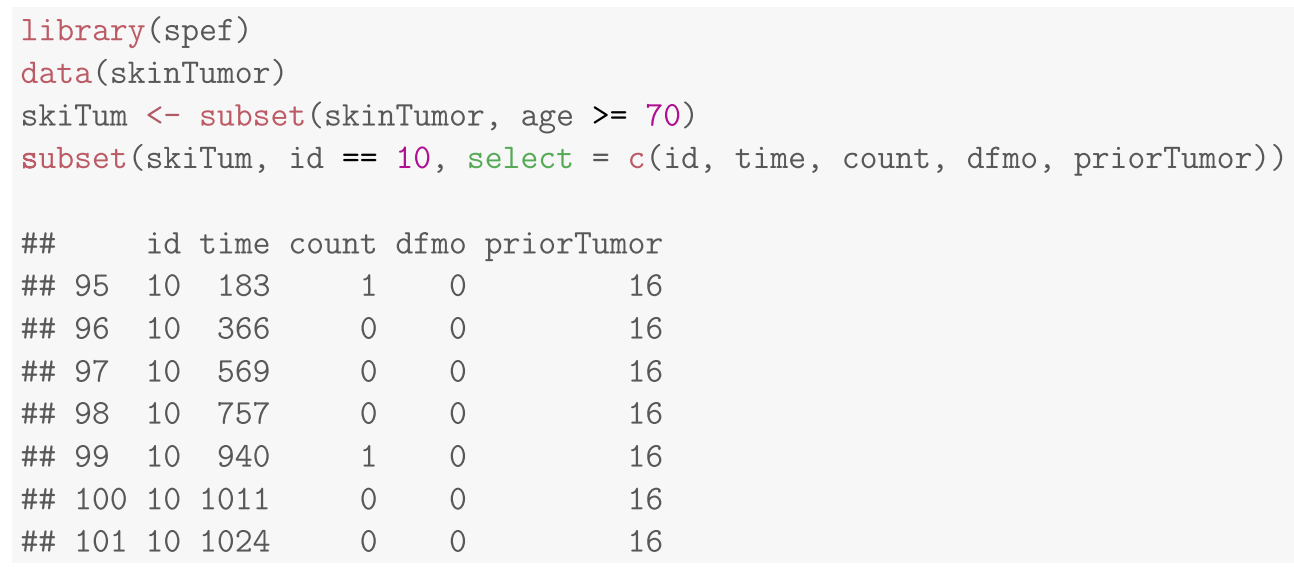

The patient with ID 10 was followed for 1024 days from the enrollment, examined seven times on days after enrollment as shown in variable time, with the corresponding number of tumours in variable count. This patient was assigned to the placebo group $(\mathrm{dfmo}=0)$ and had 16 skin tumours prior to enrollment. Treatment indicator ( $\mathrm{dfmo}$ ) and prior tumour counts (priorTumour) will be used as covariates in the regression model for the tumour occurrences in this study. Following Wang \& Yan (2011), we display the data in a tile plot that shows not only the panel count but also the examination times of each subject using package ggplot2 (Wickham, 2009):

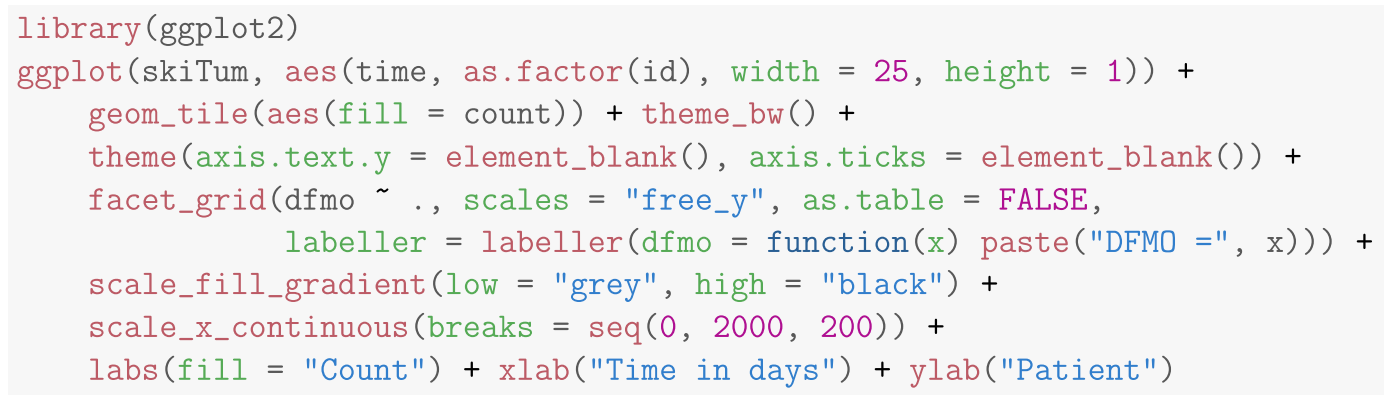

Figure 1 presents the resulting tile plot. It appears that patients in the treatment group have slightly more examinations than those in the placebo group, which might indicate informative examination times.

All the models in the sequel have the same model formula specified via Panelsurv, which is similar to the Surv function in the survival package (Therneau, 2015). We consider 


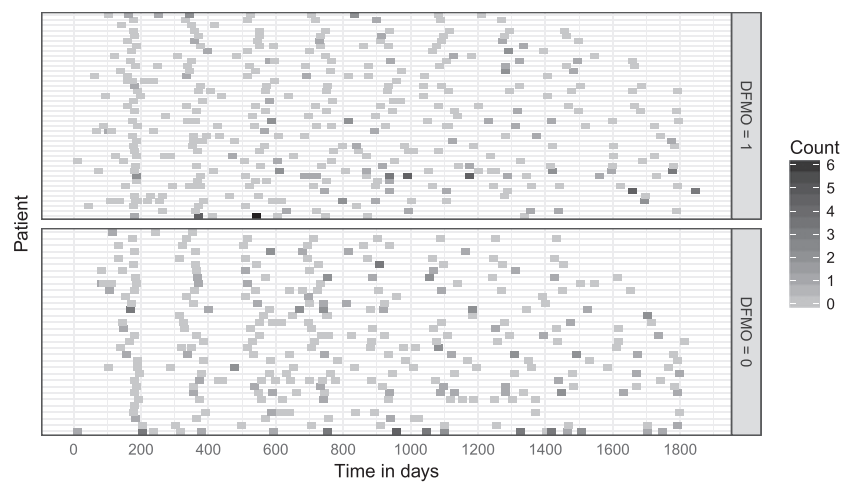

Figure 1. Tile plot of the skin tumour data. Each tile represents an examination time. Darker grays mean larger number of tumour since the last visit.

models with two covariates: dfmo and priorTumour. For better interpretation of the baseline function, we center priorTumour by its median 3:

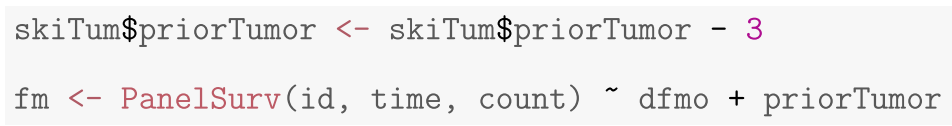

The major function to fit regression models for panel count data in the spef package is panelReg, which takes the model formula as an input and returns an object of class panelReg.

\section{Notation and Regression Models}

For subject $i, i=1, \ldots, n$, let $N_{i}(t)$ be counting process of recurrent events of interest. Suppose that the event counts are only observable at $K_{i}$ discrete random time points, $0=t_{i 0}<$ $t_{i 1}<t_{i 2}<\ldots<t_{i K_{i}} \leq \tau$, where $t_{i j}$ is the $j$ th examination time, $K_{i}$ is a positive integervalued random variable and $\tau$ is the longest follow-up time in the data. Let $G$ be the time grid formed by all distinctive examination times: $0<s_{1}<\cdots<s_{g}=\tau$, where $g$ is the number of distinctive examination times. A subject-specific, time-independent covariate vector $X_{i}$ is observed and its effect on the occurrence of the events is or primary interest. The observed data are independent and identically distributed copies of $\left\{t_{i j}, K_{i}, N_{i}\left(t_{i j}\right), X_{i} ; j=1, \ldots, K_{i}\right\}, i=$ $1, \ldots, n$. Let $n_{i j}=N_{i}\left(t_{i j}\right)-N_{i}\left(t_{i j-1}\right)$ be the number of events in the time interval $\left(t_{i j-1}, t_{i j}\right]$ and $m_{i}=N_{i}\left(Y_{i}\right)$ be the total number of events during the follow-up, where $Y_{i}=t_{i K_{i}}$ is the last examination time. Additionally, there could be a censoring or follow up time $C_{i}$, which may or may not equal to the last observation time $Y_{i}$. As in recurrent event settings, the censoring time $C_{i}$ 's are always observed unlike in the case of standard right-censored survival data. Both the examination times and the follow-up time can potentially be informative about the event process after conditioning on the covariates.

Earlier models for recurrent event processes characterise the intensity function (Gail et al., 1980; Prentice et al., 1981; Andersen \& Gill, 1982). To introduce the common models, we drop the index $i$ for ease of notation. Let $d N(t)=N\left\{(t+d t)^{-}\right\}-N\left(t^{-}\right)$. The intensity function is defined as the event occurrence rate conditional on the whole event history

$$
\lambda(t)=\lim _{\Delta \rightarrow 0+} \frac{1}{\Delta} \operatorname{Pr}\left[d N(t)=1 \mid \mathcal{H}\left(t^{-}\right)\right]
$$


where $\mathcal{H}\left(t^{-}\right)=\{N(u): 0 \leq u<t\}$ is the event history up to $t$. The Cox-type intensity model incorporates covariate $X$ in the intensity function (Andersen \& Gill, 1982)

$$
\lambda(t ; X)=\lambda_{0}(t) \exp \left(X^{\top} \beta\right),
$$

where $\lambda_{0}(t)$ is nonnegative baseline intensity function, and $\beta$ is a vector of regression coefficients for covariate vector $X$.

In practice, the Cox-type intensity model in Model (1) might be inadequate and difficult to verify (Lin et al., 2000). In contrast to Model (1), recent approaches characterise the rate function $r(t)$ of $N(t)$ defined by $E\{d N(t)\}=r(t) d t$ and the mean function $\mu(t)=\int_{0}^{t} r(s) d s$ (Nelson, 1988; Pepe \& Cai, 1993; Lawless \& Nadeau, 1995; Lin et al., 2000). Unlike the intensity function, the rate or mean function does not completely specify the stochastic nature of $N(t)$; they are, respectively, sometimes referred to as the marginal intensity and cumulative intensity function. Covariates can be incorporated in the form of proportional rates model

$$
r(t ; X)=r_{0}(t) \exp \left(X^{\top} \beta\right),
$$

for some nonnegative baseline rate function $r_{0}(t)$, or proportional means model

$$
\mu(t ; X)=\mu_{0}(t) \exp \left(X^{\top} \beta\right),
$$

for some nondecreasing baseline mean function $\mu_{0}(t)$. Since we consider time-independent covariate so far, Models (2) and (3) are equivalents.

A commonly used modification to Models (1) and (3) is to introduce a positive frailty variable or random effect. Specifically, conditional on a frailty $Z$ and covariate vector $X$, the proportional intensity model becomes

$$
\lambda(t ; X, Z)=Z \lambda_{0}(t) \exp \left(X^{\top} \beta\right)
$$

and the proportional means model becomes

$$
\mu(t ; X, Z)=Z \mu_{0}(t) \exp \left(X^{\top} \beta\right)
$$

For identification purpose, it is often assumed that $\mathrm{E}(Z \mid X)=1$. The frailty is useful in allowing over-dispersion in the count (e.g. Hua et al., 2014) or dependence between $N(\cdot)$ and the examination or censoring times (e.g. Huang et al., 2006; He et al., 2009).

The baseline intensity function $\lambda_{0}(t)$ and the baseline mean function $\mu_{0}(t)$ are often left completely unspecified and estimated nonparametrically. Since $\mu_{0}(t)$ and the cumulative baseline intensity $\Lambda_{0}(t)=\int_{0}^{t} \lambda_{0}(s) d s$ are nondecreasing functions, they can be specified by monotone splines (Ramsay, 1988). The motonone spline specification offers a good compromise between flexibility and computational advantage, so it has been adopted by many authors in various settings (Lu et al., 2009; Hua \& Zhang, 2012; Deng et al., 2015; Hua et al., 2014; Yao et al., 2016). An implementation of monotone splines is available in $\mathrm{R}$ package splines 2 for this purpose.

A recent accelerated mean model (Xu et al., 2017; Chiou et al., 2017) has rate function

$$
r(t ; X, Z)=Z r_{0}\left\{t \exp \left(X^{\top} \beta\right)\right\} \exp \left(X^{\top} \beta\right),
$$

where the distribution of frailty $Z$ is unspecified beyond $\mathrm{E}(Z \mid X)=1$. This model formulation is different from the Cox-type specifications, and it connects to the accelerated failure time models in that, unconditional on $Z, \mu(t ; X)=\mathrm{E}\{N(t) \mid X\}=\mu_{0}\left\{t \exp \left(X^{\top} \beta\right)\right\}$. The covariate effects modify the time scale of the cumulative mean function and have a direct marginal interpretation. For example, if $X$ is a treatment indicator, then the expected number of events by time $t$ among the treated subjects $(X=1)$ equals the expected number of events by time $t e^{\beta}$ in the control group $(X=0)$. 
Ramsay (1988). The same model can also be fit with PCDReg.nf function from the PCDSpl ine package (Yao \& Wang, 2014). The PCDSpl ine package further allows a gamma frailty to account for within-subject dependence (Yao et al., 2016).

A less efficient but simpler approach to obtain the regression coefficient estimate is to maximise the following pseudo-likelihood based on the Poisson distribution of each $N\left(t_{i j}\right)$ ignoring within-subject dependence

$$
L_{p}\left(\beta, \mu_{0}\right)=\sum_{i=1}^{n} \sum_{j=1}^{K_{i}} N\left(t_{i j}\right) \log \mu_{0}\left(t_{i j}\right)+N\left(t_{i j}\right) X_{i}^{\top} \beta-\mu_{0}\left(t_{i j}\right) \exp \left(X_{i}^{\top} \beta\right) .
$$

The estimator of $\beta$ with an unspecified $\mu_{0}(t)$ (Zhang, 2002) can be obtained by setting method = "MPL":

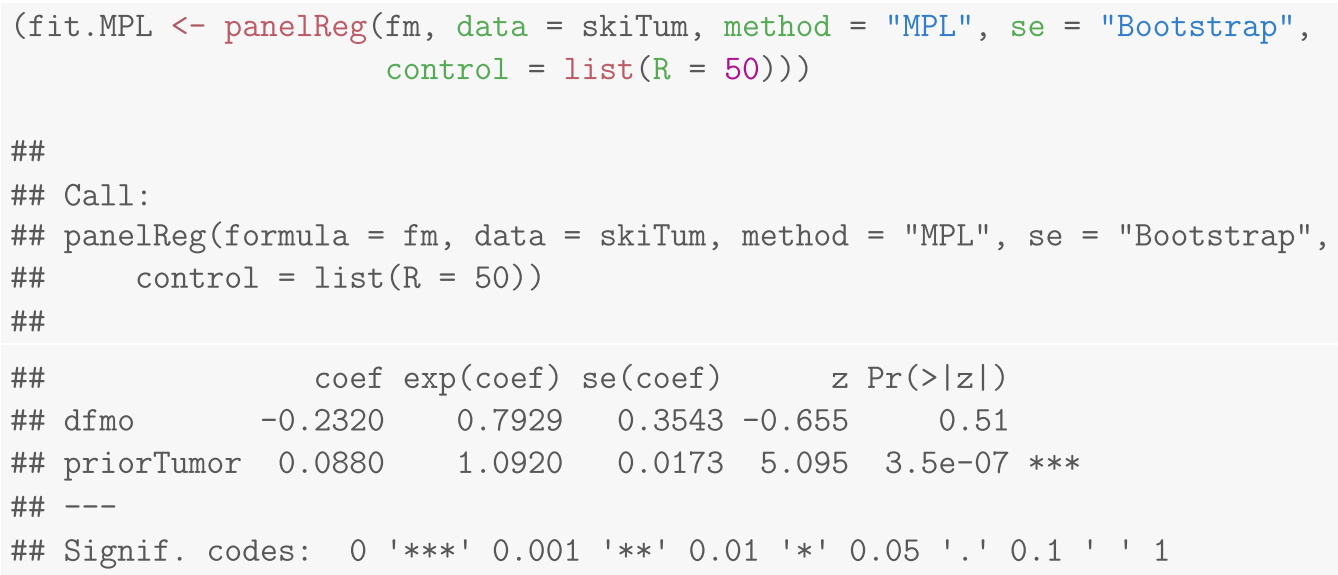

The estimator of $\beta$ when $\mu_{0}(t)$ is specified by monotone B-splines (Lu et al., 2009) can be obtained by setting method = "MPLs":

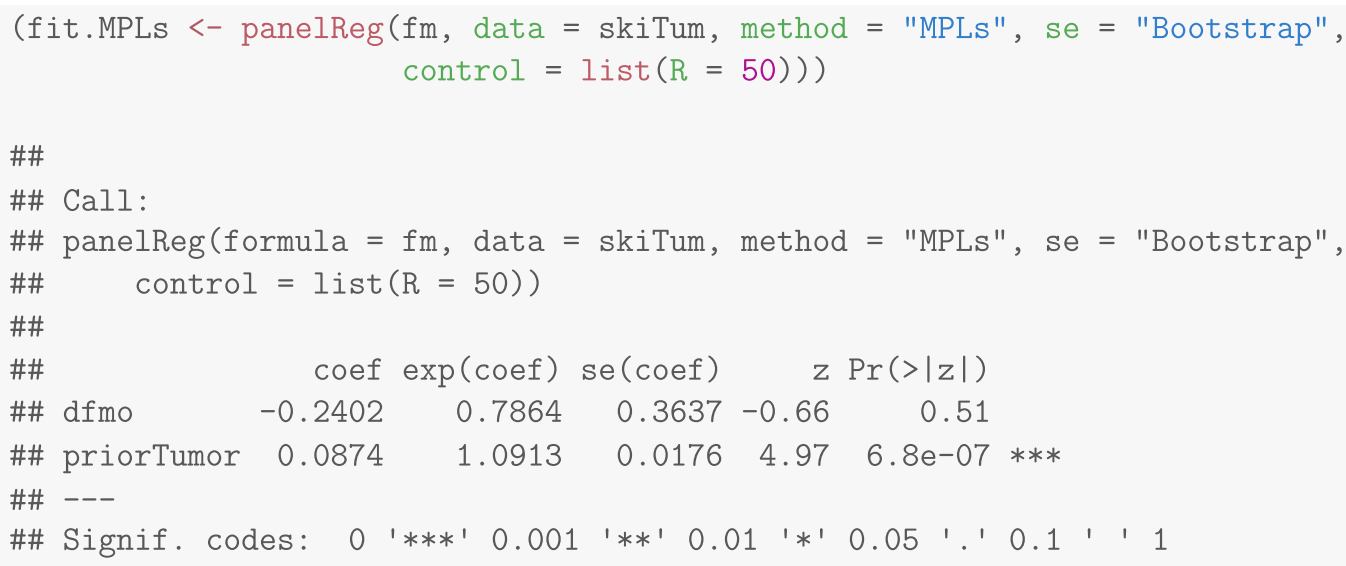

Hua et al. (2014) considered Model (4) with $Z$ assumed to be a gamma variable with mean 1 and variance $\sigma^{2}$. Under the working assumption that $N(\cdot)$ is a non-homogeneous Poisson process, the full likelihood after integrating $Z$ out has a closed-form in terms of $\beta$ and $\mu_{0}$. By approximating $\mu_{0}(t)$ with monotone splines with parameter vector $\alpha$, they estimate $\alpha$ and 
$\beta$ after fixing $\sigma^{2}$ at a method of moment estimate based on pseudolikelihood estimator from Zhang (2002) and Wellner \& Zhang (2007).

The estimated baseline mean function for the aforementioned methods can be accessed from the basel ine component in the object returned from the panelReg call. The spef package provides a utility function for its graphcial presentation through the generic function plot. For example, the estimated baseline mean function from method = "MPL" and method = "MPLS" can be plotted as follows:

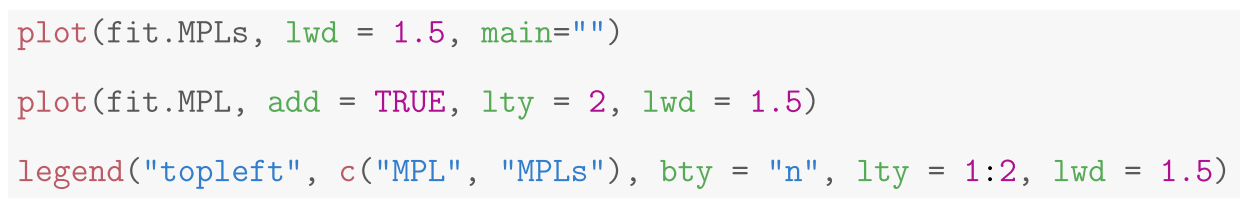

Figure 2 shows the overlaid estimated curves from the two methods. They are interpreted as the mean function for patients in the placebo group with three prior tumours. Baseline function estimates from other methods in the sequel, if available, can be a accessed similarly.

\subsection{Estimating Equation Approaches}

Sun \& Wei (2000) allow dependence among the event process, examination time process and the censoring time through covariates if the latter two follow a proportinal means model and a proportional hazards model, respectively. Define the examination time process $H_{i}(t)=\tilde{H}_{i}\left\{\min \left(t, C_{i}\right)\right\}=\sum_{j=1}^{K_{i}} I\left(t_{i j} \leq t\right)$. Assume that the mean function of $\tilde{H}_{i}(t)$ has the form

$$
\mu_{i}^{H}(t)=\mu_{0}^{H}(t) \exp \left(X_{i}^{\top} \gamma\right)
$$

where $\mu_{0}^{H}(t)$ is a completely unspecified function and $\gamma$ is a regression coefficient vector. Further assume that covariate effects on the censoring time can be specified by a Cox proportional hazards model for $C_{i}$,

$$
\lambda_{i}^{C}\left(t \mid X_{i}\right)=\lambda_{0}^{C}(t) \exp \left(X_{i}^{\top} \eta\right)
$$

where $\lambda_{0}^{C}(t)$ is a completely unspecified baseline hazard function and $\eta$ is a regression coefficient vector. The covariates are assumed to have been centred by their means in the derivation of the method.

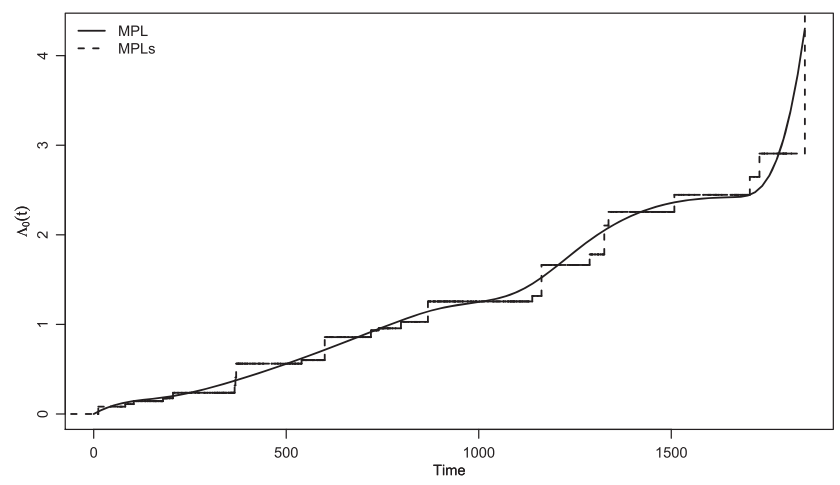

Figure 2. Estimated baseline mean function from the MPL method and the MPLs method. 
Sun $\&$ Wei (2000) proposed estimating equations by considering $\int N_{i}(t) d H_{i}(t)$. Under the model specifications for $\mu_{i}^{H}(t)$ and $\lambda_{i}^{C}(t)$,

$$
\mathrm{E}\left\{\int N_{i}(t) d H_{i}(t)\right\}=\exp \left\{X_{i}^{\top}(\beta+\gamma)\right\} \int \mu_{0}(t) S_{i}(t) d \mu_{0}^{H}(t),
$$

where $S_{i}(t)=\exp \left\{-\int_{0}^{t} \lambda_{0}^{C}(s) d s+X_{i}^{\top} \eta\right\}$. Therefore, if $\gamma$ and $\eta$ are known, $\beta$ can be estimated from the following estimating equation

$$
\sum_{i=1}^{n} X_{i} \exp \left\{-X_{i}^{\top}(\beta+\gamma)\right\} \int \frac{N_{i}(t)}{S_{i}(t)} d H_{i}(t)=0 .
$$

The unknown quantities in the equation can be replaced with their estimates: $\gamma$ can be estimated from estimating equations for proportional rates models (Lawless \& Nadeau, 1995); $\eta$ can be estimated from partial score equations (Kalbfleisch \& Prentice, 2011); and the baseline hazard $\lambda_{0}^{C}(t)$ can be estimated as in a standard survival analysis. Sun \& Wei (2000) established the consistency and asymptotic normality of the resulting estimator requiring the correct specification of the models for the examination times and the censoring time. The estimator of $\beta$ can be obtained by setting method = "EE.SWC":

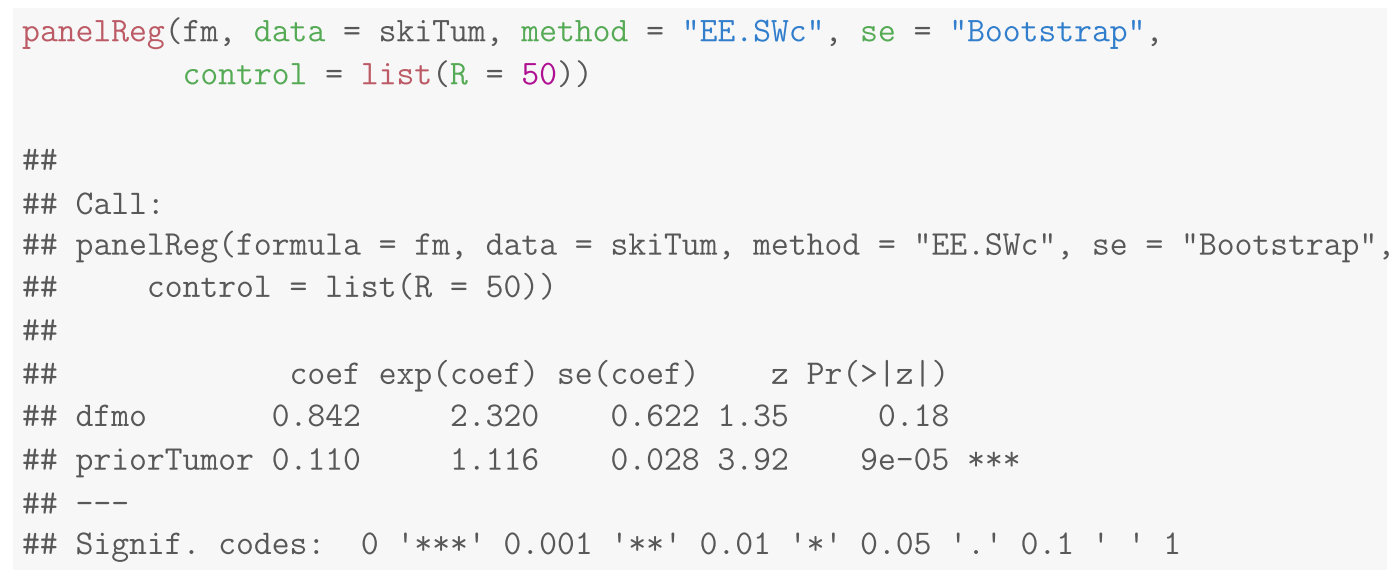

When $\eta=0$, in which case the censoring time does not depend on covariates, the estimator can be obtained by setting method = "EE.SWb":

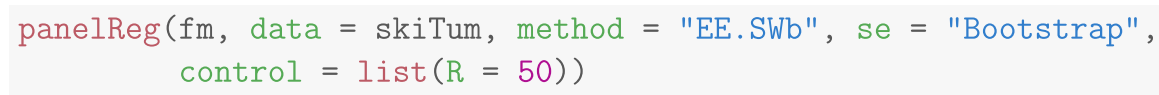

\#\# Signif. codes: 0 '***' 0.001 '**' 0.01 '*' 0.05 '.' 0.1 ' ' 1 
An even simpler version of Sun \& Wei (2000) assuming independent examination and censoring by setting $\gamma=\eta=0$ can be obtained by setting method = "EE. SWa".

$\mathrm{Hu}$ et al. (2003) proposed a more efficient estimating equation that extends the method of Lawless \& Nadeau (1995) for recurrent event analysis. Define $h_{i}(t)=H_{i}(t)-H_{i}\left(t^{-}\right)$for each $i$ so that $h_{i}(t)=1$ if $t$ is an examination time of subject $i$ and $h_{i}(t)=0$ otherwise. Assume that $E\left\{h_{i}(t)\right\}>0$ for each $t \in \mathcal{T}$ where $\mathcal{T} \subset(0, \tau]$ is the collection of all observed examination times on a grid. Conditioning on the examination times, Hu et al. (2003) proposed a natural estimating equation for $\beta$

$$
\sum_{i=1}^{n} \sum_{j=1}^{K_{i}} w\left(t_{i j}\right)\left\{X_{i}-\frac{\sum_{k=1}^{n} I\left(C_{k} \geq t_{i j}\right) X_{k} \exp \left(X_{k}^{\top} \beta\right) o_{k}\left(t_{i j}\right)}{\sum_{k=1}^{n} I\left(C_{k} \geq t_{i j}\right) \exp \left(X_{k}^{\top} \beta\right) o_{k}\left(t_{i j}\right)}\right\} n_{i j}=0,
$$

where $w(\cdot)$ is a known, possibly data dependent weight function and $o_{k}(t)$ indicates whether subject $k$ has an observation at time $t$. The estimating equation (9) was constructed under the assumption that there is more than one subject with the same examination time. Thus, this method cannot be applied to scenarios where all examination times are distinct, which implies $o_{k}\left(t_{i} j\right)=1$ when $k=i$ and 0 otherwise. Solution to the conditional estimating equations (9) with $w(t)=1$ can be obtained by setting method = "EE.HSWC":

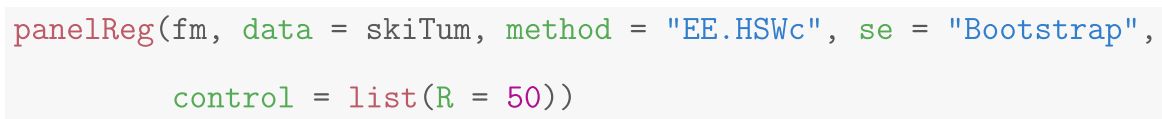

To allow covariate effects on the examination times in a proportional means Model (6), Hu et al. (2003) proposed an estimating equation unconditional on the examination times

$$
\sum_{i=1}^{n} \sum_{j=1}^{K_{i}} w\left(t_{i j}\right)\left[X_{i}-\frac{\sum_{k=1}^{n} I\left(C_{k} \geq t_{i j}\right) X_{i} \exp \left\{X_{k}^{\top}(\beta+\gamma)\right\}}{\sum_{k=1}^{n} I\left(C_{k} \geq t_{i j}\right) \exp \left\{X_{k}^{\top}(\beta+\gamma)\right\}}\right] n_{i j}=0,
$$

where $\gamma$ needs to be replaced with an estimate as in solving (8). In contrast to (8), this equation does not require model specification of the censoring time. See Section 5.4.3 of Sun \& Zhao (2013) for more discussion on comparison of the estimating equation approaches. Solution to the marginal estimating equations (10) can be obtained by setting method = "EE.HSWm": 


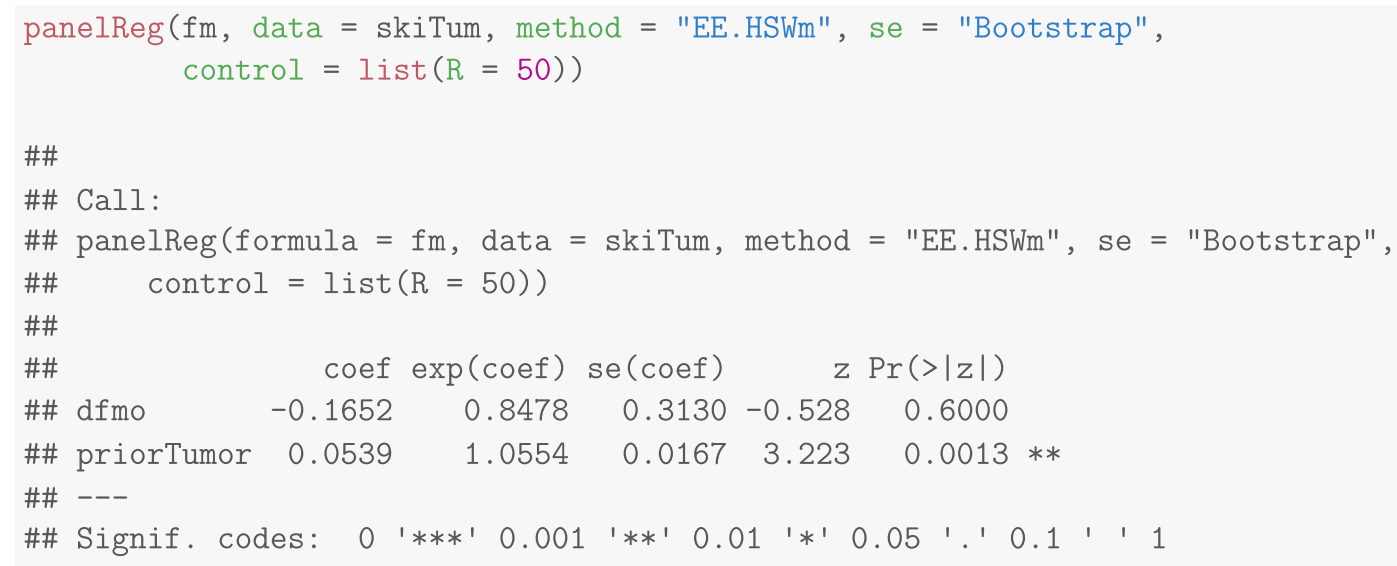

Because panel counts are similar to longitudinal data, Hua \& Zhang (2012) applied generalised estimating equations (Liang \& Zeger, 1986) to marginal Model (3) with $\log \lambda(t)$ approximated by monotone splines with parameters $\alpha$ as in Lu et al. (2009). The panel counts from subject $i$ form a vector $\mathcal{N}_{i}=\left\{N_{i}\left(t_{i 1}\right), \ldots, N_{i}\left(t_{i, K_{i}}\right)\right\}^{\top}$, with mean vector $\mu_{i}=\left\{\mu\left(t_{i 1} ; X_{i}\right), \ldots, \mu\left(t_{i K_{i}} ; X_{i}\right)\right\}^{\top}$. The generalised estimating equation has the form

$$
\sum_{i=1}^{n} \frac{\partial \mu_{i}^{\top}}{\partial \theta} V_{i}^{-1}\left(\mathcal{N}_{i}-\mu_{i}\right)=0,
$$

where $\theta^{\top}=\left(\beta^{\top}, \alpha^{\top}\right)$, and $V_{i}$ is a $K_{i} \times K_{i}$ working covariance matrix of $\mathcal{N}_{i}$. Hua \& Zhang (2012) used a two-iterative algorithm to solve for $\theta$. First, a Newton-Raphson update is applied to solve (11); second, the estimate of $\alpha$ is projected to a legitimate space via quadratic programming such that the resulting splines is monotone nondecreasing. Flexible choices of the working covariance matrix $V_{i}$ 's can lead to higher efficiency in estimation and robustness to overdispersion.

\section{Informative Examination/Censoring Times}

\subsection{Frailty Methods}

One way to allow informative examination times after conditioning on covariates is to introduce a frailty, or random effect that is shared by both the recurrent event process and the examination time process. Huang et al. (2006) considered Model (4), which allows the examination times to be associated with the event process through the frailty after conditioning on the covariates. The approach of Huang et al. (2006) is especially appealing in that there is no need to specify the distribution of the frailty, or models for the examination process and the censoring time. The estimation procedure takes advantage of the fact that, conditional on $\left\{Z_{i}, X_{i}, K_{i}, Y_{i}\right\}$, the unobserved $K_{i}$ examination times are order statistics of independent and identically distributed random variables with distribution function

$$
F_{i}(t)=\frac{\mu\left(t ; X_{i}, Z_{i}\right)}{\mu\left(Y_{i} ; X_{i}, Z_{i}\right)}=\frac{\mu_{0}(t)}{\mu_{0}\left(Y_{i}\right)} .
$$

This formulation suggests that the estimation of $F(t)$ does not involve $X_{i}$ and $Z_{i}$. Let $\Phi(t)=$ $\mu_{0}(t) / \mu_{0}(\tau)$, where $\tau$ is still the longest follow-up time. A nonparametric estimator of $F(t)$ is obtained by maximising 


$$
\prod_{i=1}^{n} \prod_{j=1}^{K_{i}}\left[\frac{\Phi\left(T_{i, j}\right)-\Phi\left(T_{i, j-1}\right)}{\Phi\left(Y_{i}\right)}\right]^{n_{i j}},
$$

which is mathematically equivalent to the likelihood constructed from a set of independently interval-censored and right-truncated data. Therefore, the maximisation of the likelihood can be implemented by the Turnbull's self-consistency algorithm (Turnbull, 1976). When computational performance is of concern, the squared extrapolation method of Varadhan \& Roland (2008) can be adopted to accelerate the maximisation. Then, $\Lambda(\tau)$ and $\beta$ are obtained from solving

$$
n^{-1} \sum_{i=1}^{n} w_{i}\left(\begin{array}{c}
1 \\
X_{i}
\end{array}\right)\left[m_{i} \Phi\left(Y_{i}\right)^{-1}-\mu_{0}(\tau) \exp \left(X_{i}^{\top} \beta\right)\right]=0,
$$

where $w_{i}$ is a weight function and $\Phi(\cdot)$ is replaced with its estimate. This approach with $w_{i}=1$ is requested by setting method = "HWZ":

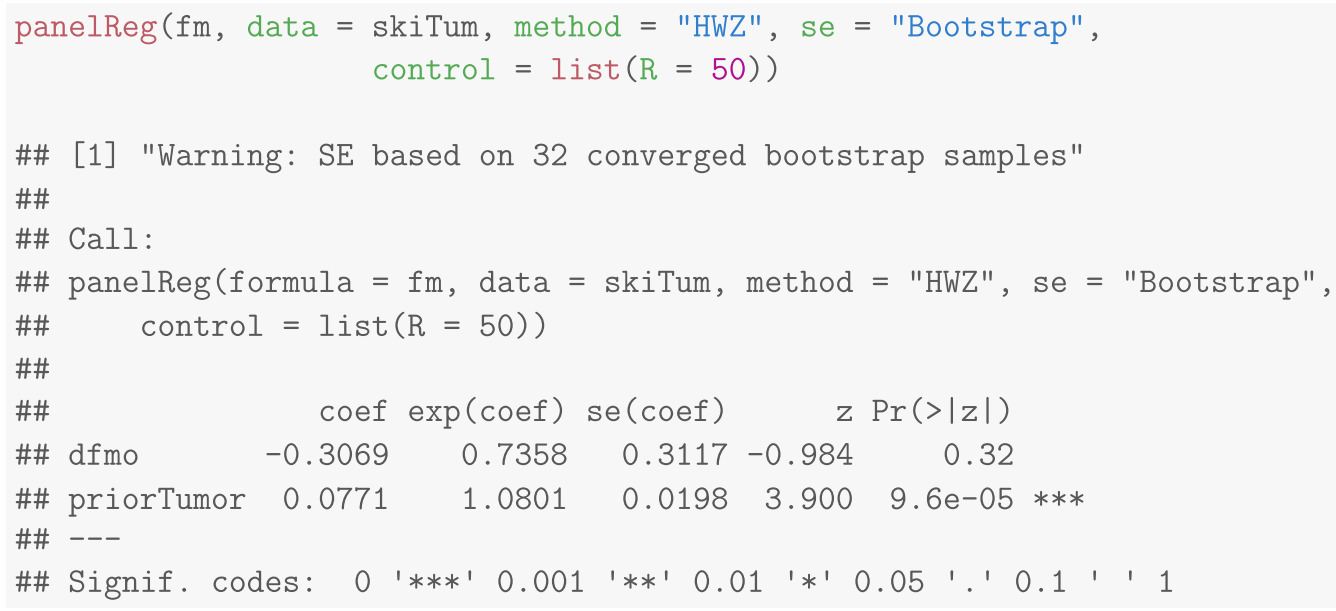

A warning message indicates that not all of the 50 bootstrap converged. The reported bootstrap standard errors are based on those that converged.

Alternative approaches specify models for the examination times and the censoring time. Extending the estimation strategies of Sun \& Wei (2000), Sun et al. (2007) investigated a similar semiparametric model with $Z_{i}^{\alpha}$ in place of $Z_{i}$ in Model (4), where $Z_{i}$ is an unobserved multiplicative frailty introduced into Model (6) for the examination times. He et al. (2009) used two frailties to introduce dependence among the three Models (3), (6) and (7) beyond covariate effects. Specifically, one frailty enters all three models while the other enters Models (3) and (7). Model parameters are estimated through a three-step estimation procedure. This method imposes a distributional assumption on the underlying random effect and requires the examination process to be a nonhomogeneous Poisson process, which is needed in an EM algorithm in handling the parameters and frailties in the model for the examination process. Zhao et al. (2013) proposed a more general model which replaces $Z$ in Model (4) with $f(Z)$, where $Z$ is a multiplicative frailty introduced into Model (6) as in Sun et al. (2007), and $f$ is a positive, completely unspecified link function. They relaxed the Poisson assumption for the examination process. The methods of He et al. (2009) and Zhao et al. (2013) are presented in detail in Sun and Zhao (2013, Sections 6.2-6.3).

\subsection{Augmented Estimating Equations}

Wang et al. (2013) approached the problem by treating the unobserved event times as missing data. Consider the time grid $G$ in Section 3 , let $\mathbb{N}_{i j}=N_{i}\left(s_{j}\right)-N_{i}\left(s_{j-1}\right)$ be the number of 
events occurred in $\left(s_{j-1}, s_{j}\right]$. Only summations of $\mathbb{N}_{i j}$ 's over those subintervals whose union coincides with an observation window are observed. Regardless of the examination times, if $\mathbb{N}_{i j}$ 's were observed, under conditional independent censoring, Model (3) suggests a set of complete-data estimating equations:

$$
\begin{aligned}
\sum_{i=1}^{n}\left[\mathbb{N}_{i j}-\lambda_{j} \exp \left(X_{i}^{\top} \beta\right)\right] r_{i j} & =0, \quad j=1, \ldots, G, \\
\sum_{i=1}^{n} \sum_{j=1}^{G}\left[\mathbb{N}_{i j}-\lambda_{j} \exp \left(X_{i}^{\top} \beta\right)\right] X_{i} r_{i j} & =0,
\end{aligned}
$$

where $\lambda_{j}=\Lambda\left(s_{j}\right)-\Lambda\left(s_{j-1}\right)$ is the baseline mean number of events occurring in interval $\left(s_{j-1}, s_{j}\right]$, and $r_{i j}=I\left(s_{j} \leq C_{i}\right)$ is the at-risk indicator. The model parameters are estimated by an Expectation-Solving algorithm (Elashoff \& Ryan, 2004), an analog of the EM algorithm for estimating equations without specifying the full likelihood. The algorithm iterates between imputing the values of $\mathbb{N}_{i j}$ 's and solving the conditional expected version of the complete-data estimating equations given the observed data. This method is called by setting method = "AEE":

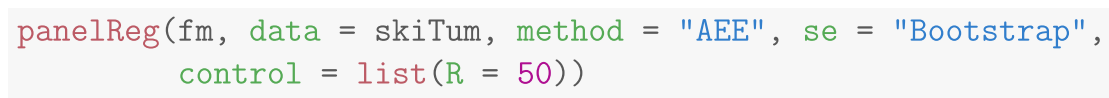

In the case of informative censoring, the number of events between the last examination time $Y_{i}$ and $\tau$ is also treated as missing and imputed using a working model; see (Wang et al., 2013) for more details. This method is requested by setting method = "AEEX":

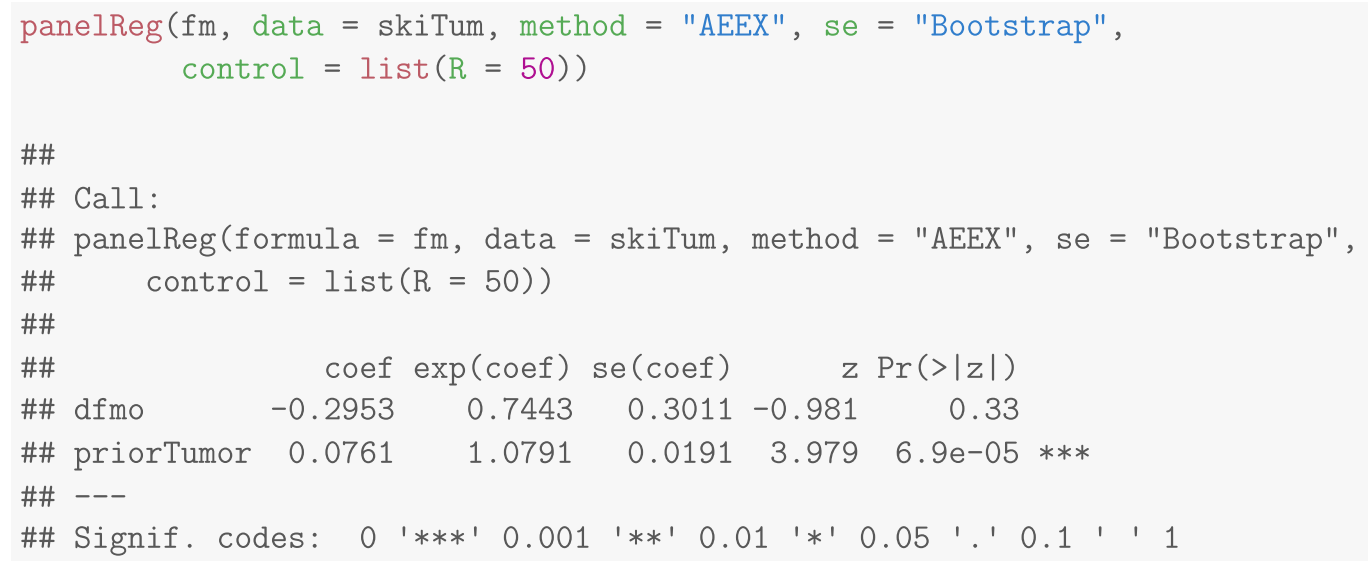




\subsection{Accelerated Mean Model}

Chiou et al. (2017) estimated the parameters of the accelerated mean model (5) by a profile estimating equation approach. Specifically, consider the transformed times $t_{i j}^{*}(\beta)=$ $t_{i j} \exp \left(X_{i}^{\top} \beta\right)$ and censoring time $Y_{i}^{*}(\beta)=Y_{i} \exp \left(X_{i}^{\top} \beta\right), i=1, \ldots, n$. Conditional on $\left(Z_{i}, X_{i}, K_{i}, Y_{i}\right)$, the unobserved $K_{i}$ examination times on the transformed scale $t_{i j}^{*}(\beta)$ are order statistics of independent and identically distributed random variables with distribution function $\mu_{0}(t) / \mu_{0}\left(Y_{i}^{*}(\beta)\right)$. Let $\Phi(t)=\mu_{0}(t) / \mu_{0}\left(\tau_{\beta}\right)$, where $\tau_{\beta}=\tau \sup _{i} \exp \left(X_{i}^{\top} \beta\right)$. For given $\beta, \Phi$ can be estimated with the same method of Huang et al. (2006) except that the estimate depends on $\beta$. Define $\hat{\Phi}_{n}(t ; \beta)$ as the resulting estimator. Then, $\beta$ is estimated by solving the estimating equation

$$
\sum_{i=1}^{n} X_{i}\left[m_{i} \hat{\Phi}_{n}^{-1}\left\{Y_{j}^{*}(\beta) ; \beta\right\}-\frac{1}{n} \sum_{j=1}^{n} m_{j} \hat{\Phi}_{n}^{-1}\left\{Y_{j}^{*}(\beta) ; \beta\right\}\right]=0
$$

In our implementation, this equation is solved with a gradient-free spectral method (Barzilai \& Borwein ; 1988;La Cruz et al. ; 2006). The accelerated mean model is called by setting method = "AMM". Because fitting this model is much more computing intensive than other methods, we timed this call:

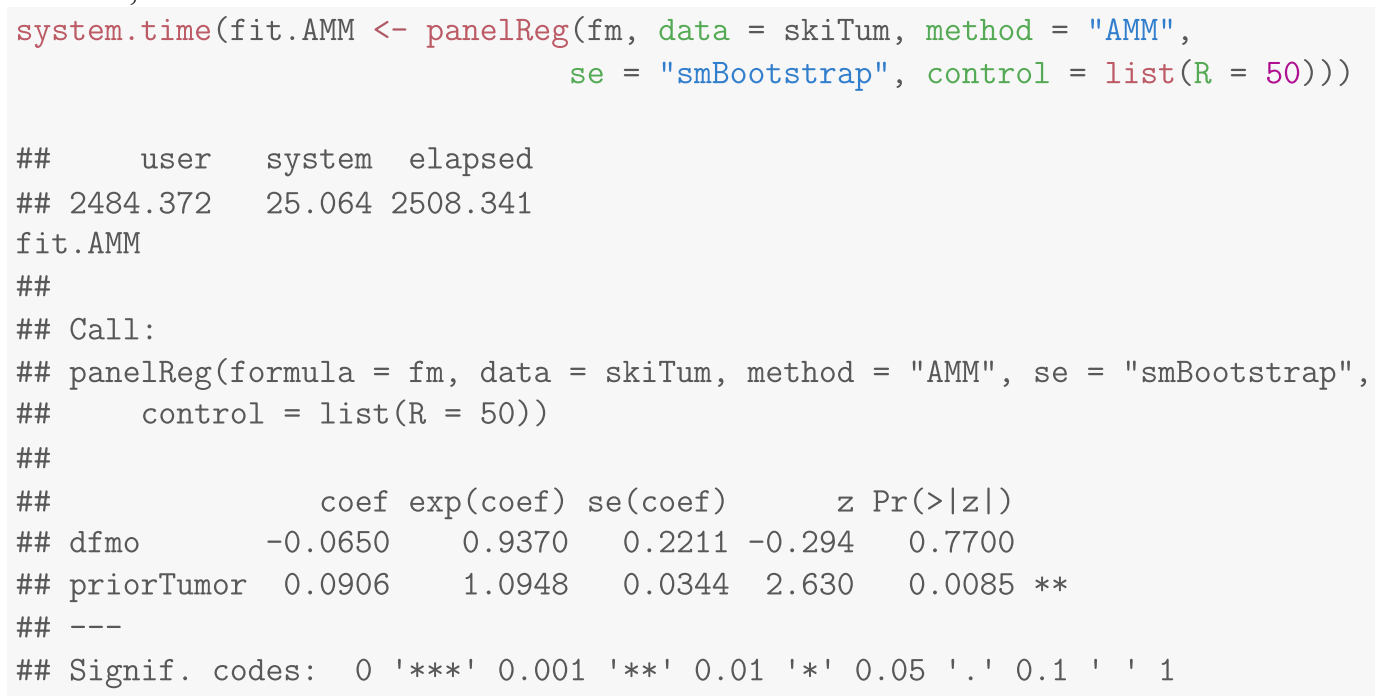

The standard errors was obtained from a smoothed bootstrap procedure proposed in Chiou et al. (2017) by setting se = "smBootstrap". The standard bootstrap procedure to obtain the standard errors is still available by setting se = "Bootstrap".

\section{Simulation}

We extended the simulation studies in Huang et al. (2006) and Wang et al. (2013) to provide a thorough comparison among the estimators discussed in this paper. Because the regression coefficient in the accelerated mean model is interpreted differently than those in the proportional means model, we focus here on the comparison of the regression coefficient estimates in the proportional means model. We generated recurrent events from a Poisson process with mean model specified in Model (4) for $t \in[0, \tau]$ with $\tau=10$. The baseline mean function 
Table 1. Simulation results for Scenario 1 where the examination times and the recurrent events are independent with $n=100$. Column bias is the average bias; ESE is the empirical standard error; ASE is the average standard error based on the standard bootstrap; $C P$ is the empirical coverage probability (\%); time is the average time in seconds used in both point estimation and bootstrap variance estimation.

\begin{tabular}{|c|c|c|c|c|c|c|c|c|c|}
\hline & \multicolumn{2}{|l|}{ bias } & \multicolumn{2}{|l|}{ ESE } & \multicolumn{2}{|l|}{ ASE } & \multicolumn{2}{|c|}{$\mathrm{CP}(\%)$} & \multirow[t]{2}{*}{ Time } \\
\hline & $\beta_{1}$ & $\beta_{2}$ & $\beta_{1}$ & $\beta_{2}$ & $\beta_{1}$ & $\beta_{2}$ & $\beta_{1}$ & $\beta_{2}$ & \\
\hline & \multicolumn{9}{|c|}{$Z=1$} \\
\hline MLs & -0.001 & 0.001 & 0.037 & 0.019 & 0.038 & 0.021 & 95.3 & 96.9 & 588.7 \\
\hline MPL & -0.001 & 0.002 & 0.043 & 0.023 & 0.043 & 0.024 & 94.9 & 96.5 & 237.9 \\
\hline MPLs & -0.001 & 0.001 & 0.042 & 0.023 & 0.044 & 0.023 & 96.3 & 96.4 & 543.9 \\
\hline EE.SWC & -0.007 & 0.005 & 0.205 & 0.101 & 0.202 & 0.103 & 96.2 & 96.6 & 72.1 \\
\hline EE.SWb & 0.005 & 0.006 & 0.149 & 0.087 & 0.159 & 0.087 & 96.1 & 96.1 & 47.7 \\
\hline EE.SWa & 0.005 & 0.007 & 0.129 & 0.078 & 0.137 & 0.076 & 96.4 & 95.2 & 3.4 \\
\hline EE.HSWm & -0.005 & 0.011 & 0.241 & 0.135 & 0.242 & 0.128 & 95.2 & 93.8 & 65.6 \\
\hline HWZ & -0.001 & 0.001 & 0.046 & 0.022 & 0.046 & 0.023 & 94.0 & 93.8 & 1227.9 \\
\hline $\mathrm{AEE}$ & -0.001 & 0.002 & 0.037 & 0.019 & 0.039 & 0.021 & 95.7 & 96.8 & 176.3 \\
\hline \multirow[t]{2}{*}{$A E E X$} & -0.002 & -0.002 & 0.044 & 0.021 & 0.046 & 0.024 & 95.1 & 95.8 & 375.4 \\
\hline & \multicolumn{9}{|c|}{$Z \sim$ gamma distribution } \\
\hline MLs & 0.007 & -0.007 & 0.206 & 0.126 & 0.195 & 0.107 & 94.7 & 90.3 & 676.0 \\
\hline MPL & 0.010 & -0.007 & 0.215 & 0.127 & 0.198 & 0.107 & 93.8 & 90.4 & 264.8 \\
\hline MPLs & 0.009 & -0.007 & 0.216 & 0.129 & 0.202 & 0.110 & 93.8 & 90.3 & 578.4 \\
\hline EE.SWC & -0.002 & 0.010 & 0.310 & 0.148 & 0.297 & 0.148 & 94.0 & 95.9 & 66.3 \\
\hline EE.SWb & 0.012 & 0.007 & 0.216 & 0.113 & 0.227 & 0.121 & 95.5 & 96.0 & 43.7 \\
\hline EE.SWa & 0.004 & 0.007 & 0.205 & 0.108 & 0.210 & 0.113 & 94.9 & 96.4 & 3.0 \\
\hline EE.HSWm & 0.013 & -0.010 & 0.304 & 0.179 & 0.310 & 0.166 & 95.9 & 92.8 & 60.9 \\
\hline HWZ & 0.007 & -0.007 & 0.201 & 0.124 & 0.190 & 0.113 & 93.3 & 91.3 & 1053.2 \\
\hline $\mathrm{AEE}$ & 0.007 & -0.007 & 0.205 & 0.125 & 0.194 & 0.110 & 94.8 & 91.2 & 237.1 \\
\hline \multirow[t]{2}{*}{$A E E X$} & -0.005 & -0.011 & 0.200 & 0.122 & 0.192 & 0.112 & 94.6 & 91.5 & 362.5 \\
\hline & \multicolumn{9}{|c|}{$Z \sim$ uniform distribution } \\
\hline MLs & -0.008 & -0.005 & 0.177 & 0.106 & 0.171 & 0.096 & 94.1 & 90.6 & 674.3 \\
\hline MPL & -0.009 & -0.008 & 0.183 & 0.110 & 0.175 & 0.097 & 94.4 & 90.8 & 266.5 \\
\hline MPLs & -0.008 & -0.007 & 0.187 & 0.118 & 0.179 & 0.099 & 95.2 & 90.2 & 581.7 \\
\hline EE.SWC & -0.006 & 0.004 & 0.305 & 0.139 & 0.274 & 0.139 & 92.4 & 95.6 & 66.8 \\
\hline EE.SWb & -0.007 & 0.001 & 0.195 & 0.111 & 0.210 & 0.112 & 96.3 & 95.1 & 44.1 \\
\hline EE.SWa & -0.014 & 0.002 & 0.174 & 0.105 & 0.194 & 0.103 & 97.0 & 94.9 & 3.1 \\
\hline EE.HSWm & 0.002 & 0.001 & 0.308 & 0.176 & 0.297 & 0.163 & 94.7 & 93.4 & 61.3 \\
\hline HWZ & -0.010 & -0.005 & 0.177 & 0.109 & 0.164 & 0.098 & 92.6 & 90.1 & 1070.1 \\
\hline $\mathrm{AEE}$ & -0.009 & -0.005 & 0.176 & 0.111 & 0.169 & 0.095 & 93.7 & 90.4 & 235.0 \\
\hline$A E E X$ & -0.013 & -0.009 & 0.173 & 0.107 & 0.168 & 0.095 & 94.6 & 90.1 & 366.7 \\
\hline
\end{tabular}

was set to be $\mu_{0}(t)=2 t$. Two mutually independent covariates, $X_{i 1}$ and $X_{i 2}$, were generated from the Bernoulli distribution with rate 0.5 and the standard normal distribution, respectively. The regression coefficients were set to be $\beta=\left(\beta_{1}, \beta_{2}\right)^{\top}=(0.5,1)^{\top}$. The subject-specific frailty $Z_{i}$ had three configurations: (a) fixed at constant 1; (b) generated from a gamma distribution with mean 1 and variance 0.5 ; or (c) generated from a uniform distribution over [0,2]. The sample size $n$ had two levels, 100 and 200 .

We considered three scenarios depending on how examination times associate with recurrent events:

- Scenario 1: Examination times and recurrent events are independent. The number of examinations, $K_{i}$, was generated from a district uniform distribution on $\{1, \ldots, 6\}$; and the distinct examination times, $t_{i 1}, \ldots, t_{i K_{i}}$, were the order statistics of $K_{i}$ independent and identically distributed uniform distribution over $[0,10]$. 
- Scenario 2: Examination times and recurrent events are independent conditioning on the covariates. If $X_{i 1} X_{i 2}>0$, then the number of examinations, $K_{i}$, was generated from a district uniform distribution on $\{1, \ldots, 8\}$ and the distinct examination times were the order statistics of $K_{i}$ independent and identically distributed exponential distribution with mean 2; otherwise, $K_{i}$ and $t_{i 1}, \ldots, t_{i} K_{i}$ were generated in the same fashion as in Scenario 1.

- Scenario 3: Examination times are informative about the recurrent events after conditioning on the covariates. If $X_{i 1} X_{i 2}>0$ and $Z_{i}>1$, then $K_{i}$ and $t_{i 1}, \ldots, t_{i} K_{i}$ were generated as in the case of $X_{i 1} X_{i 2}>0$ in Scenario 2; otherwise, they were generated in the same fashion as in Scenario 1.

Under the study designs, Scenario 3 reduces to Scenario 1 when $Z_{i}$ was fixed at 1 , but the two scenarios are different otherwise. In Scenario 2 when the examination times and recurrent events are independent conditioning on covariate, subjects with $X_{i 1}=1$ and $X_{i 2}>0$ are

Table 2. Simulation results for Scenario 2 where the examination times and the recurrent events are conditionally independent given covariates with $n=100$. Column bias is the average bias; ESE is the empirical standard error; ASE is the average standard error based on the standard bootstrap; CP is the empirical coverage probability (\%); time is the average time in seconds used in both point estimation and bootstrap variance estimation.

\begin{tabular}{|c|c|c|c|c|c|c|c|c|c|}
\hline & \multicolumn{2}{|l|}{ bias } & \multicolumn{2}{|l|}{ ESE } & \multicolumn{2}{|l|}{ ASE } & \multicolumn{2}{|c|}{ CP $(\%)$} & \multirow[t]{2}{*}{ Time } \\
\hline & $\beta_{1}$ & $\beta_{2}$ & $\beta_{1}$ & $\beta_{2}$ & $\beta_{1}$ & $\beta_{2}$ & $\beta_{1}$ & $\beta_{2}$ & \\
\hline & \multicolumn{9}{|c|}{$Z=1$} \\
\hline MLs & -0.001 & 0.001 & 0.043 & 0.022 & 0.045 & 0.023 & 96.0 & 96.4 & 600.9 \\
\hline MPL & -0.002 & 0.002 & 0.052 & 0.026 & 0.053 & 0.028 & 95.1 & 96.7 & 243.4 \\
\hline MPLs & -0.004 & 0.001 & 0.050 & 0.025 & 0.052 & 0.027 & 95.8 & 96.5 & 599.5 \\
\hline EE.SWC & -0.229 & -0.193 & 0.372 & 0.164 & 0.362 & 0.158 & 79.1 & 68.5 & 65.8 \\
\hline EE.SWb & -0.833 & -0.347 & 0.195 & 0.097 & 0.197 & 0.105 & 1.7 & 8.9 & 43.5 \\
\hline EE.SWa & -0.338 & -0.139 & 0.147 & 0.080 & 0.149 & 0.081 & 39.7 & 59.6 & 3.1 \\
\hline EE.HSWm & -1.527 & -0.399 & 0.291 & 0.164 & 0.292 & 0.158 & 0.0 & 29.8 & 60.8 \\
\hline HWZ & -0.008 & 0.000 & 0.060 & 0.028 & 0.059 & 0.029 & 94.6 & 95.7 & 1120.5 \\
\hline $\mathrm{AEE}$ & -0.001 & 0.001 & 0.043 & 0.022 & 0.045 & 0.024 & 95.2 & 96.5 & 236.7 \\
\hline \multirow[t]{2}{*}{$A E E X$} & -0.020 & -0.007 & 0.055 & 0.027 & 0.056 & 0.029 & 94.3 & 96.8 & 474.5 \\
\hline & \multicolumn{9}{|c|}{$Z \sim$ gamma distribution } \\
\hline MLs & 0.010 & -0.007 & 0.202 & 0.119 & 0.192 & 0.104 & 94.4 & 90.9 & 728.1 \\
\hline MPL & 0.025 & -0.002 & 0.200 & 0.122 & 0.195 & 0.105 & 94.5 & 90.9 & 282.0 \\
\hline MPLs & 0.004 & -0.006 & 0.203 & 0.124 & 0.199 & 0.108 & 94.7 & 90.5 & 684.1 \\
\hline EE.SWC & -0.277 & -0.202 & 0.464 & 0.207 & 0.405 & 0.187 & 79.3 & 71.4 & 66.9 \\
\hline EE.SWb & -0.828 & -0.346 & 0.247 & 0.127 & 0.256 & 0.134 & 10.5 & 27.1 & 43.9 \\
\hline EE.SWa & -0.333 & -0.139 & 0.216 & 0.110 & 0.221 & 0.115 & 68.1 & 77.5 & 3.1 \\
\hline EE.HSWm & -1.500 & -0.409 & 0.349 & 0.207 & 0.340 & 0.198 & 1.5 & 40.4 & 61.4 \\
\hline HWZ & 0.010 & -0.006 & 0.212 & 0.128 & 0.198 & 0.115 & 92.9 & 91.7 & 1081.3 \\
\hline $\mathrm{AEE}$ & 0.014 & -0.006 & 0.199 & 0.119 & 0.190 & 0.103 & 94.4 & 90.9 & 341.3 \\
\hline \multirow[t]{2}{*}{ AEEX } & -0.007 & -0.016 & 0.203 & 0.123 & 0.192 & 0.108 & 94.8 & 90.8 & 512.6 \\
\hline & \multicolumn{9}{|c|}{$Z \sim$ gamma distribution } \\
\hline MLs & -0.007 & 0.001 & 0.177 & 0.109 & 0.168 & 0.094 & 94.5 & 91.1 & 673.2 \\
\hline MPL & 0.014 & 0.007 & 0.185 & 0.110 & 0.171 & 0.093 & 94.8 & 90.4 & 264.8 \\
\hline MPLs & -0.003 & 0.004 & 0.187 & 0.112 & 0.176 & 0.098 & 94.9 & 90.7 & 638.4 \\
\hline EE.SWC & -0.272 & -0.192 & 0.442 & 0.205 & 0.392 & 0.188 & 80.2 & 75.4 & 61.7 \\
\hline EE.SWb & -0.808 & -0.345 & 0.239 & 0.128 & 0.242 & 0.128 & 8.8 & 25.4 & 40.9 \\
\hline EE.SWa & -0.327 & -0.138 & 0.202 & 0.103 & 0.204 & 0.109 & 64.6 & 77.1 & 3.5 \\
\hline EE.HSWm & -1.492 & -0.400 & 0.349 & 0.209 & 0.332 & 0.183 & 0.8 & 41.2 & 57.3 \\
\hline HWZ & -0.017 & 0.001 & 0.179 & 0.117 & 0.172 & 0.094 & 93.0 & 89.7 & 989.9 \\
\hline$A E E$ & -0.002 & 0.002 & 0.174 & 0.108 & 0.166 & 0.090 & 94.5 & 89.5 & 301.4 \\
\hline AEEX & -0.030 & -0.009 & 0.168 & 0.112 & 0.169 & 0.096 & 95.0 & 90.2 & 463.4 \\
\hline
\end{tabular}


Table 3. Simulation results for Scenario 3 where the examination times are informative about the recurrent events after conditioning on covariates with $n=100$. Column bias is the average bias; ESE is the empirical standard error; ASE is the average standard error based on the standard bootstrap; $C P$ is the empirical coverage probability (\%); time is the average time in seconds used in both point estimation and bootstrap variance estimation.

\begin{tabular}{|c|c|c|c|c|c|c|c|c|c|}
\hline & \multicolumn{2}{|l|}{ bias } & \multicolumn{2}{|l|}{ ESE } & \multicolumn{2}{|l|}{ ASE } & \multicolumn{2}{|c|}{ CP $(\%)$} & \multirow[t]{2}{*}{ Time } \\
\hline & $\beta_{1}$ & $\beta_{2}$ & $\beta_{1}$ & $\beta_{2}$ & $\beta_{1}$ & $\beta_{2}$ & $\beta_{1}$ & $\beta_{2}$ & \\
\hline & \multicolumn{9}{|c|}{$Z \sim$ gamma distribution } \\
\hline MLs & -0.147 & -0.038 & 0.199 & 0.117 & 0.188 & 0.102 & 86.7 & 87.7 & 594.9 \\
\hline MPL & -0.164 & -0.048 & 0.210 & 0.115 & 0.190 & 0.101 & 84.5 & 87.3 & 221.6 \\
\hline MPLs & -0.172 & -0.048 & 0.208 & 0.118 & 0.192 & 0.103 & 85.5 & 86.6 & 496.9 \\
\hline EE.SWC & -0.248 & -0.110 & 0.318 & 0.153 & 0.290 & 0.147 & 81.6 & 85.8 & 64.9 \\
\hline EE.SWb & -0.379 & -0.155 & 0.215 & 0.113 & 0.225 & 0.119 & 59.9 & 74.7 & 42.6 \\
\hline EE.SWa & -0.206 & -0.078 & 0.192 & 0.100 & 0.208 & 0.109 & 86.1 & 88.7 & 2.9 \\
\hline EE.HSWm & -0.723 & -0.191 & 0.314 & 0.186 & 0.309 & 0.166 & 38.5 & 70.6 & 58.8 \\
\hline HWZ & -0.003 & -0.006 & 0.212 & 0.125 & 0.199 & 0.112 & 91.7 & 91.2 & 990.9 \\
\hline $\mathrm{AEE}$ & -0.144 & -0.038 & 0.198 & 0.116 & 0.189 & 0.100 & 88.8 & 90.7 & 225.6 \\
\hline \multirow[t]{2}{*}{$A E E X$} & -0.015 & -0.014 & 0.206 & 0.122 & 0.191 & 0.105 & 92.6 & 89.9 & 419.1 \\
\hline & \multicolumn{9}{|c|}{$Z \sim$ uniform distribution } \\
\hline MLS & -0.143 & -0.032 & 0.181 & 0.113 & 0.173 & 0.096 & 86.7 & 89.7 & 633.3 \\
\hline MPL & -0.169 & -0.039 & 0.186 & 0.114 & 0.177 & 0.097 & 83.3 & 88.8 & 246.3 \\
\hline MPLs & -0.175 & -0.040 & 0.189 & 0.115 & 0.180 & 0.099 & 83.0 & 89.3 & 547.7 \\
\hline EE.SWC & -0.258 & -0.130 & 0.318 & 0.150 & 0.292 & 0.147 & 79.1 & 83.2 & 64.7 \\
\hline EE.SWb & -0.453 & -0.183 & 0.218 & 0.104 & 0.216 & 0.115 & 45.2 & 66.5 & 43.9 \\
\hline EE.SWa & -0.221 & -0.087 & 0.185 & 0.099 & 0.193 & 0.103 & 80.6 & 87.9 & 3.1 \\
\hline EE.HSWm & -0.861 & -0.229 & 0.323 & 0.179 & 0.303 & 0.165 & 21.0 & 65.7 & 59.8 \\
\hline HWZ & -0.008 & 0.001 & 0.181 & 0.123 & 0.171 & 0.112 & 93.4 & 91.5 & 1010.1 \\
\hline$A E E$ & -0.142 & -0.032 & 0.181 & 0.112 & 0.171 & 0.105 & 86.6 & 90.6 & 241.7 \\
\hline$A E E X$ & -0.022 & -0.010 & 0.174 & 0.116 & 0.167 & 0.097 & 94.6 & 90.9 & 434.9 \\
\hline
\end{tabular}

more likely to be examined more frequently. In Scenario 3 when the examination times are informative about the recurrent events, the design implies a positive association between the underlying recurrent event process and the examination time process; subjects with $X_{i 1}=1$, $X_{i 2}>0$ and $Z_{i}>1$ have a higher event rate and tend to be examined more frequently. Since examination times were generated from continuous probability distributions for all three scenarios, EE.HSWC estimator was excluded from the study as the EE.HSWC estimator is not applicable to scenarios when there are no ties in examination times. The standard errors were estimated using the standard bootstrap procedure by setting se = "Bootstrap" with $\mathrm{R}=$ 200 bootstrap samples. For each configuration, 1000 datasets were generated and analysed. The timing results were obtained on a Linux machine with $2 \mathrm{GHz}$ CPU.

Table 1 presents the results under Scenario 1. All estimators are virtually unbiased. The empirical standard errors and the estimated standard errors from the standard bootstrap procedure agree closely for all estimators, suggesting that the bootstrap procedure provides valid inference. The estimating-equation-based estimators were fastest to compute, but they appear to have higher standard errors than other estimators. All estimators had higher standard errors in the case of gamma frailty, which has high variance than the case of uniform frailty. The empirical coverage percentages are mostly reasonably close to the nominal level of $95 \%$, with a closer agreement with larger sample size (results for $n=200$ not shown).

Table 2 summarises the results under Scenario 2. No estimator except those based on estimating equations show noticeable bias. The substantial bias and, consequently, the low coverage 
rate of the confidence intervals from the estimating equation approaches are due to their misspecification of the examination time process. The other estimators do not require specification of the examination time process, which might not be of primary interest. They appear to have similar results regarding bias and standard errors. Among them, the AEE estimator is the fastest and has the smallest standard errors, albeit the advantage in standard error is small.

Table 3 summarises the results under Scenario 3. Under this setting of informative examination times, the only unbiased estimators appear to be the HWZ estimator and the AEEX estimator, with comparable standard errors. This is explained by the rationals on which they are derived. Their coverage rates of the confidence intervals were a bit lower than the nominal rate for the continuous regression coefficient, and the agreement improves as the sample size becomes $n=200$ (results not shown). The AEEX estimator is twice as fast as the HWZ estimator.

\section{Discussion}

Nonparametric estimation of the mean cumulative function or mean rate function (e.g. Sun \& Zhao, 2013, Chapters 3 and 4) plays an important role in many methods for semiparametric regression models. Estimation of semiparametric approaches often involves an alternate iteration between updating the estimate of $\beta$ and updating the estimate of $\mu_{0}(t)$, the latter of which is often based on nonparametric estimation given $\beta$. For example, the MLE and MPLE of Wellner \& Zhang (2007) are based on the one-sample nonparametric MLE and MPLE of Wellner \& Zhang (2000). The method of Huang et al. (2006) does not require alternate iteration in estimating the parameters of Model (4) because of the special structure of this model. When the idea is adapted to the accelerated mean Model (5) of Chiou et al. (2017), nonparametric estimation given the parametric part becomes necessary in an alternate iteration procedure. Some nonparametric estimation methods with self-consistent algorithm (Hu et al., 2009a, 2009b) have not been but could be combined with a parametric estimation procedure to form a semiparametric approach. For methods implemented in the spef package, nonparametric estimation can be requested by setting right hand of the model formula to be intercept only; for example, Panelsurv (id, time, count) 1. In addition, the baseline function estimates can be plotted with the generic plot function as illustrated in Sections 4 and 5 .

The scope of this review is limited to available implementations of semiparametric regression models with time-independent covariates. A wide range of topics on panel count data have been studied, many of which have been reviewed by Sun \& Zhao (2013). Examples are nonparametric comparison (Zhang, 2006), semiparametric transformation models (Li et al., 2010), multivariate panel count data analysis (He et al., 2008; Li et al., 2011; Zhang et al., 2013; Li et al., 2015), measurement errors (Kim, 2007), mixed recurrent event and panel count data analysis (Zhu et al., 2013), varying-coefficient models (He et al., 2017), incorporation of observation history in regression (Li et al., 2010; Deng et al., 2015) and so on. Some topics are worth investigating; for example, adapting the semiparametric regression with time-dependent covariates for recurrent event data (Huang et al., 2010) to panel count data. The unavailability of cutting-edge methods to practitioners calls for user-friendly, quality controlled software implementation as reproductive statistical research gains sharpened focus.

\section{References}

Andersen, P. K. \& Gill, R. D. (1982). Cox's regression model for counting processes: A large sample study. Ann. Statist., 10, 1100-1120. 
Bailey, H. H., Kim, K., Verma, A. K., Sielaff, K., Larson, P. O., Snow, S., Lenaghan, T., Viner, J. L., Douglas, J. \& Dreckschmidt, N. E. (2010). A randomized, double-blind, placebo-controlled phase 3 skin cancer prevention study of $\alpha$-difluoromethylornithine in subjects with previous history of skin cancer. Cancer Prev. Res., 3(1), 35-47.

Barzilai, J. \& Borwein, J. M. (1988). Two-point step size gradient methods. IMA J. Numer. Anal., 8(1), $141-148$.

Chiou, S. H., Wang, X. \& Yan, J. (2017). spef: Semiparametric estimating functions. R package version 1.0-6.

Chiou, S., Xu, G., Yan, J. \& Huang, C.-Y. (2017). Semiparametric estimation of the accelerated mean model with panel count data under informative examination times. Biometrics. To appear. https://doi.org/10.1111/biom.12840.

Croissant, Y., Millo, G. et al. (2008). Panel data econometrics in R: The plm package, 27(2), 1-43.

Deng, S., Liu, L. \& Zhao, X. (2015). Monotone spline-based least squares estimation for panel count data with informative observation times. Biom. J., 57(5), 743-765.

Elashoff, M. \& Ryan, L. (2004). An EM algorithm for estimating equations. J. Comput. Graph. Statist., 13(1), $48-65$.

Gail, M. H., Santner, T. J. \& Brown, C. C. (1980). An analysis of comparative carcinogenesis experiments based on multiple times to tumor. Biometrics, 36, 255-266.

He, X., Feng, X., Tong, X. \& Zhao, X. (2017). Semiparametric partially linear varying coefficient models with panel count data. Lifetime Data Anal., 23(3), 439-466.

He, X., Tong, X. \& Sun, J. (2009). Semiparametric analysis of panel count data with correlated observation and follow-up times. Lifetime Data Anal., 15(2), 177-196.

He, X., Tong, X., Sun, J. \& Cook, R. J. (2008). Regression analysis of multivariate panel count data. Biostatistics, 9(2), 234-248.

Hsiao, C. (2014). Analysis of Panel Data. New York: Cambridge University Press.

Hu, X. J., Lagakos, S. W. \& Lockhart, R. A. (2009). Generalized least squares estimation of the mean function of a counting process based on panel counts. Statist. Sinica, 19, 561.

Hu, X. J., Lagakos, S. W. \& Lockhart, R. A. (2009). Marginal analysis of panel counts through estimating functions. Biometrika, 96(2), 445-456.

Hu, X., Sun, J. \& Wei, L.-J. (2003). Regression parameter estimation from panel counts. Scand. J. Stat., 30(1), $25-43$.

Hua, L. \& Zhang, Y. (2012). Spline-based semiparametric projected generalized estimating equation method for panel count data. Biostatistics, 13(3), 440-454.

Hua, L., Zhang, Y. \& Tu, W. (2014). A spline-based semiparametric sieve likelihood method for over-dispersed panel count data. Canad. J. Statist., 42(2), 217-245.

Huang, C.-Y., Qin, J. \& Wang, M.-C. (2010). Semiparametric analysis for recurrent event data with time-dependent covariates and informative censoring. Biometrics, 66(1), 39-49.

Huang, C.-Y., Wang, M.-C. \& Zhang, Y. (2006). Analysing panel count data with informative observation times. Biometrika, 93(4), 763-775.

Kalbfleisch, J. \& Lawless, J. F. (1985). The analysis of panel data under a markov assumption. J. Amer. Statist. Assoc., 80(392), 863-871.

Kalbfleisch, J. D. \& Prentice, R. L. (2011). The statistical analysis of failure time data. New Jersey: John Wiley \& Sons.

Kim, Y.-J. (2007). Analysis of panel count data with measurement errors in the covariates. J. Stat. Comput. Simul., 77(2), 109-117.

La Cruz, W., Martínez, J. \& Raydan, M. (2006). Spectral residual method without gradient information for solving large-scale nonlinear systems of equations. J. Stat. Comput. Simul., 75(255), 1429-1448.

Lawless, J. F. \& Nadeau, C. (1995). Some simple robust methods for the analysis of recurrent events. Technometrics, 37(2), 158-168.

Li, Y., He, X., Wang, H., Zhang, B. \& Sun, J. (2015). Semiparametric regression of multivariate panel count data with informative observation times. J. Multivariate Anal., 140, 209-219.

Li, N., Park, D.-H., Sun, J. \& Kim, K. (2011). Semiparametric transformation models for multivariate panel count data with dependent observation process. Canad. J. Statist., 39(3), 458-474.

Li, N., Sun, L. \& Sun, J. (2010). Semiparametric transformation models for panel count data with dependent observation process. Statistics in Biosciences, 2(2), 191-210.

Liang, K.-Y. \& Zeger, S. L. (1986). Longitudinal data analysis using generalized linear models. Biometrika, 73, 1322.

Lin, D., Wei, L., Yang, I. \& Ying, Z. (2000). Semiparametric regression for the mean and rate functions of recurrent events. J. R. Stat. Soc. Ser. B, 62(4), 711-730.

Lu, M., Zhang, Y. \& Huang, J. (2009). Semiparametric estimation methods for panel count data using monotone b-splines. J. Amer. Statist. Assoc., 104(487), 1060-1070.

Ma, L. \& Sundaram, R. (2018). Analysis of gap times based on panel count data with informative observation times and unknown start time. J. Amer. Statist. Assoc., 113(521), 294-305.

Nelson, W. (1988). Graphical analysis of system repair data. Journal of Quality Technology, 20(1), 24-35. 
Pepe, M. S. \& Cai, J. (1993). Some graphical displays and marginal regression analyses for recurrent failure times and time dependent covariates. J. Amer. Statist. Assoc., 88(423), 811-820.

Prentice, R. L., Williams, B. J. \& Peterson, A. V. (1981). On the regression analysis of multivariate failure time data. Biometrika, 68(2), 373-379.

R Core Team. (2017). R: A Language and Environment for Statistical Computing. Vienna, Austria: R Foundation for Statistical Computing.

Ramsay, J. O. (1988). Monotone regression splines in action. Statist. Sci., 3, 425-441.

Riphahn, R. T., Wambach, A. \& Million, A. (2003). Incentive effects in the demand for health care: A bivariate panel count data estimation. J. Appl. Econometrics, 18(4), 387-405.

Sun, J., Tong, X. \& He, X. (2007). Regression analysis of panel count data with dependent observation times. Biometrics, 63(4), 1053-1059.

Sun, J. \& Wei, L. J. (2000). Regression analysis of panel count data with covariate-dependent observation and censoring times. J. R. Stat. Soc. Ser. B, 62(2), 293-302.

Sun, J. \& Zhao, X. (2013). Statistical analysis of panel count data. New York: Springer.

Therneau, T. M. (2015). A package for survival analysis in $S$. version 2.38 .

Turnbull, B. W. (1976). The empirical distribution function with arbitrarily grouped, censored and truncated data. $J$. R. Stat. Soc. Ser. B, 38(3), 290-295.

Varadhan, R. \& Roland, C. (2008). Simple and globally convergent methods for accelerating the convergence of any EM algorithm. Scand. J. Stat., 35(2), 335-353.

Wang, X., Ma, S. \& Yan, J. (2013). Augmented estimating equations for semiparametric panel count regression with informative observation times and censoring time. Statist. Sinica, 23, 359-381.

Wang, X. \& Yan, J. (2011). Fitting semiparametric regressions for panel count survival data with an R package spef. Computer Methods and Programs in Biomedicine, 104(2), 278-285.

Wellner, J. A. \& Zhang, Y. (2000). Two estimators of the mean of a counting process with panel count data. Ann. Statist., 28, 779-814.

Wellner, J. A. \& Zhang, Y. (2007). Two likelihood-based semiparametric estimation methods for panel count data with covariates. Ann. Statist., 35(5), 2106-2142.

Wickham, H. (2009). ggplot2: Elegant Graphics for Data Analysis. New York: Springer-Verlag.

Xu, G., Chiou, S. H., Huang, C.-Y., Wang, M.-C. \& Yan, J. (2017). Joint scale-change models for recurrent events and failure time. J. Amer. Statist. Assoc., 112, 794-805.

Yao, B. \& Wang, L. (2014). PCDSpline: Semiparametric regression analysis of panel count data using monotone splines. R package version 1.0 .

Yao, B., Wang, L. \& He, X. (2016). Semiparametric regression analysis of panel count data allowing for within-subject correlation. Comput. Stat. Data Anal., 97, 47-59.

Zhang, Y. (2002). A semiparametric pseudolikelihood estimation method for panel count data. Biometrika, 89(1), $39-48$.

Zhang, Y. (2006). Nonparametric k-sample tests with panel count data. Biometrika, 93(4), 777-790.

Zhang, H., Zhao, H., Sun, J., Wang, D. \& Kim, K. (2013). Regression analysis of multivariate panel count data with an informative observation process. J. Multivariate Anal., 119, 71-80.

Zhao, X., Tong, X. \& Sun, J. (2013). Robust estimation for panel count data with informative observation times. Comput. Stat. Data Anal., 57(1), 33-40.

Zhu, L., Tong, X., Zhao, H., Sun, J., Srivastava, D. K., Leisenring, W. \& Robison, L. L. (2013). Statistical analysis of mixed recurrent event data with application to cancer survivor study. Stat. Med., 32(11), 1954-1963.

[Received August 2017, accepted April 2018] 